\title{
Carrier-phase inter-frequency biases of GLONASS receivers
}

\author{
Lambert Wanninger
}

Received: 6 January 2011 / Accepted: 15 July 2011 / Published online: 2 August 2011

(c) The Author(s) 2011. This article is published with open access at Springerlink.com

\begin{abstract}
The frequency division multiplexing of the GLONASS signals causes inter-frequency biases in the receiving equipment. These biases vary considerably for receivers from different manufacturers and thus complicate or prevent carrier-phase ambiguity fixing. Complete and reliable ambiguity fixing requires a priori information of the carrier-phase inter-frequency bias differences of the receivers involved. GLONASS carrier-phase inter-frequency biases were estimated for 133 individual receivers from 9 manufacturers. In general, receivers of the same type and even receivers from the same manufacturer show similar biases, whereas the differences among manufacturers can reach up to $0.2 \mathrm{~ns}$ (more than $5 \mathrm{~cm}$ ) for adjacent frequencies and thus up to $2.4 \mathrm{~ns}(73 \mathrm{~cm})$ for the complete $\mathrm{L} 1$ or L2 frequency bands. A few individual receivers were identified whose inter-frequency biases behave differently as compared to other receivers of the same type or whose biases vary with time.
\end{abstract}

Keywords GLONASS · Carrier-phase · Inter-frequency bias $\cdot$ Ambiguity fixing

\section{Introduction}

The present GLONASS navigation system uses frequency division multiplexing (FDMA-frequency division multiple access) to make the signals from individual satellites distinguishable. This results in the use of several adjacent frequencies for the broadcast signals in the two frequency bands L1 and L2. In both frequency bands, each GLONASS satellite transmits its signal on one of 14 frequencies. Antipodal

L. Wanninger $(\varangle)$

Geodätisches Institut, TU Dresden, 01062, Dresden, Germany

e-mail: lambert.wanninger@tu-dresden.de satellites share the same frequency, so that a full satellite constellation of 24 GLONASS satellites can be used (Hofmann-Wellenhof et al. 2008; ICD 2008). In the coming years, new signals will use code division multiplexing like GPS (Revnivykh 2010). But for the next decade or so, only GLONASS FDMA-signals will provide continuous dual-frequency coverage.

As a consequence of this FDMA approach, different hardware biases exist in GLONASS receiving channels even within one frequency band. For this reason and also because of the various signal wavelengths, GLONASS carrier-phase processing differs from that of GPS. In general, older receiving equipment of the same type has experienced quite similar inter-frequency biases, and these may be removed to a large extent in differential mode (Zinoviev 2005). For many years, only a few manufacturers offered geodetic GPS/GLONASS equipment and furthermore, they took care that the fractional parts of the inter-frequency biases of old and new receiver types were quite similar (Zinoviev et al. 2009). Thus GLONASS carrier-phase ambiguity fixing could easily be performed between receivers of different brands.

However, since 2006 more and more manufacturers entered the GPS/GLONASS receiver market. They seem to be paying less attention to forcing the GLONASS carrierphase inter-frequency biases of the new equipment to be the same as or similar to the biases of older equipment or equipment from other manufacturers. Thus, today GLONASS receivers from different manufacturers show large differences in their inter-frequency biases.

Pratt et al. (1998) proved for a pair of Ashtech Z-18 receivers that their GLONASS individual frequency dependent delays are a linear function of frequency. This finding was confirmed for several receiver types by Wanninger and Wallstab-Freitag (2007). They showed that biases between receivers from different manufacturers and between adjacent 
frequency channels can differ in the order of $0.1 \mathrm{~ns}(3 \mathrm{~cm})$. The maximum signal delay difference between lowest and highest frequency channels can thus exceed the signal wavelength.

If not modeled properly, these GLONASS inter-frequency biases can prevent correct and reliable carrier-phase ambiguity fixing in baselines with receivers from different manufacturers. Furthermore, if the differential biases are large, successful ambiguity fixing in mixed baselines requires the application of a priori corrections for these biases. After ambiguity-fixing, the precise values of these linear biases must be estimated together with the baseline coordinate solution. A priori values of linear biases for several receiver types were published in Wanninger and Wallstab-Freitag (2007). They were confirmed by Zinoviev et al. (2009).

The analysis of GLONASS carrier-phase inter-frequency biases by Wanninger and Wallstab-Freitag (2007) was based on data observed over short or zero (two receivers sharing one antenna) baselines. Only about ten individual receivers were used to estimate the differential biases. A much larger sample is necessary to confirm that these inter-frequency biases agree among receivers of the same type or among many receivers from a single manufacturer. Furthermore, since 2007 some more manufacturers released GPS/GLONASS equipment. Since the manufacturers do not publish differential interfrequency biases for their new receiver types in relation to older receiver types, it is crucial to obtain a priori bias values for real-time kinematic (RTK) and other precise geodetic applications which require GLONASS ambiguity fixing.

In this paper, GLONASS differential carrier-phase interfrequency biases are estimated and analyzed for 133 individual GPS/GLONASS receivers, comprising 19 receiver types produced by 9 different manufacturers. It is shown how large the differential biases are between receivers from different manufacturers, how well individual receiver biases agree for each receiver type, and how stable these biases are over time. Finally, the paper includes an updated list of a priori corrections for the differential biases for receivers from all nine manufacturers.

This paper does not deal with receiver inter-frequency biases of the GLONASS code observations (Kozlov et al. 2000; Yamanda et al. 2010), since they seem to have different characteristics and they are of less importance for geodetic GNSS applications based on carrier-phase observations.

\section{Determination of inter-frequency biases}

Determination of GLONASS carrier-phase inter-frequency biases is explained and performed best when using singledifference carrier-phase observations. When calculating single-differences of simultaneous carrier-phase observations of two receivers to one satellite, satellite clock errors and instrumental biases are eliminated and orbit errors, ionospheric and tropospheric effects are greatly reduced. Double-differences, i.e. differences between simultaneous carrier-phase observations of two receivers and two satellites, which are often used with GPS, should be avoided with GLONASS because of the satellite-specific carrier wavelengths. More details on single-difference and double-difference GNSS observations can be found in e.g. Leick (2004) or Hofmann-Wellenhof et al. (2008).

Since all GLONASS carrier-phase data used in this work originates from GPS/GLONASS receivers, and since a combined processing of GPS/GLONASS data was performed, two different observation equations are required, one for GPS and one for GLONASS. Single difference carrier-phase observations $\Delta \Phi(\mathrm{m})$ can be described as:

$$
\begin{aligned}
& \Delta \Phi_{a, b}^{\mathrm{GPS}, i}=\Delta R_{a, b}^{i}+c_{0} \cdot \Delta \delta t_{a, b}^{\mathrm{GPS}}+\lambda \cdot \Delta N_{a, b}^{i}+\varepsilon_{\Delta \Phi} \\
& \Delta \Phi_{a, b}^{\mathrm{GLONASS}, i}=\Delta R_{a, b}^{i}+c_{0} \\
& \cdot\left(\Delta \delta t_{a, b}^{\mathrm{GLONASS}}+k^{i} \cdot \Delta \delta h_{a, b}^{\mathrm{GLONASS}}\right)+\lambda \cdot \Delta N_{a, b}^{i}+\varepsilon_{\Delta \Phi}
\end{aligned}
$$

where subscripts $a, b$ stand for the stations involved, the superscripts GPS and GLONASS indicate the GNSS satellite system, and the superscript $i$ specifies the individual satellite. $\Delta R(\mathrm{~m})$ is the single-difference of the satellite-receiver ranges, $c_{0}(\mathrm{~m} / \mathrm{s})$ is the speed of light in a vacuum, $\Delta \delta t$ (s) is the difference of the receiver clocks which depends on the satellite system due to different receiver hardware delays, $\triangle \delta h^{G L O N A S S}$ (s) is the difference between the interfrequency biases for two receivers on adjacent GLONASS frequencies, $k(-)$ is the GLONASS channel number, $\lambda(\mathrm{m})$ is the signal wavelength, $\Delta N(-)$ is the single-difference of the carrier phase ambiguity and $\varepsilon_{\Delta \Phi}(\mathrm{m})$ is the sum of all uncorrected systematic and random errors in the single-difference observable.

In order to remove the singularity between the receiver clock term and the ambiguities, one $\Delta N$ must be fixed to an arbitrary value, allowing it to be removed from the list of parameters. For GPS, this procedure is essentially identical to form double-difference observations. For GLONASS however, forming double-difference observations is not recommended because of the various signal wavelengths.

Another singularity exists between the GLONASS ambiguities $\Delta N$ and the linearly modeled inter-frequency biases $\Delta \delta h$. If all ambiguities are to be estimated (float solution), $\Delta \delta h$ cannot be estimated simultaneously. Only after fixing of at least one additional single-difference ambiguity $\Delta N$, the system of equations becomes non-singular and $\Delta \delta h$ can be estimated.

In practice, a priori values of $\Delta \delta h$ are introduced in order to remove a large portion of the inter-frequency biases. Then, a first GLONASS $\Delta N$ is fixed to an arbitrary value to remove 
the singularity with the receiver clock term. Now, the first estimation of all other ambiguities can be performed without solving for $\Delta \delta h$ and, if at least one ambiguity can be fixed to its true value, the system of equations becomes non-singular. In subsequent processing steps, the remaining ambiguities $\Delta N$ can be estimated along-side the inter-frequency biases $\Delta \delta h$.

This approach is realized by the baseline processing engine Wa1 including the combined ambiguity fixing for both GPS and GLONASS observations. Since independent receiver clock errors are estimated for GPS and GLONASS (cf. Eqs. 1 and 2), there is a certain separation between the ambiguities of both systems. Nevertheless, all ambiguities are estimated and fixed on equal terms.

In the context of this paper, only the estimates of the interfrequency biases for adjacent GLONASS frequencies $\Delta \delta h$ are of interest. They can either be estimated with GLONASS carrier-phase observations only or they can be estimated in a combined GPS/GLONASS data processing. We performed combined processing since the increased amount of observational data enlarges the percentage of fixed ambiguities.

In subsequent parts of this paper, estimated inter-frequency biases are presented in units of meters. To achieve this, $\Delta \delta h$ values (s) were multiplied with the speed of light in a vacuum $c_{0}(\mathrm{~m} / \mathrm{s})$.

\section{Observation data sets}

The ideal data set to determine GLONASS inter-frequency biases would consist of observations from many receivers of various receiver types employed at the same site. The short baselines mean that ionospheric and tropospheric effects would almost completely cancel out by differencing, and thus very accurate inter-frequency biases could be estimated. Due to the high costs of collecting such data, it was decided to use already existing data instead. In the archives of continuously operating GNSS reference station networks, data sets from GPS/GLONASS receivers have become quite common in recent years. These data sets have the disadvantage that much longer inter-station distances have to be accepted and thus some ionospheric and tropospheric refraction effects will influence the results. In order to include a large number of receivers and receiver types in this study, while at the same time keeping the maximum baseline lengths well below $1,000 \mathrm{~km}$, only sites across the European continent were selected. Here, a high density of GPS/GLONASS receivers with publicly available data exists. Furthermore, as these GPS/GLONASS receivers come from various manufacturers, these data sets are very well suited for this study.

Two weeks during 2010, GPS week 1570 (7-13 February 2010) and GPS week 1599 (29 August-4 September 2010), were selected to have a large sample size.
Intentionally, they are separated by half a year to check or disprove eventual temperature effects on the GLONASS inter-frequency biases. For those three stations with detected larger variations of the GLONASS inter-frequency biases between these 2 weeks, longer data samples were analyzed (cf. Fig. 8).

Table 1 gives an overview of the number of receivers used in the 2 weeks. The stations are shown in Figs. 1 and 2. Most of the stations belong to the EUREF Permanent Network (EPN, http://www.epncb.oma.be, Bruyninx et al. 2001) or the network of the International GNSS Service (IGS, http://www.igs.org, Dow et al. 2009). A few more stations were added to analyze an additional receiver type and to increase the number of receivers for one manufacturer. Two of these additional stations are part of the French TERIA network (http://www.reseau-teria.com). They are equipped with Ashtech Pro Flex 500 CORS receivers. Data from stations equipped with Javad receivers were made available by the Bavarian Commission for International Geodesy, Munich, Germany, and by the Institute of Communications and Navigation of the German Aerospace Center, Neustrelitz, Germany.

Data from eight European GPS/GLONASS stations from the EPN and IGS networks could not be processed successfully and are not included in Table 1; Figs. 1, or 2. Most of these stations, although equipped with GPS/GLONASS receivers, in fact collect no or only very few GLONASS observations. Data from other stations were not available for more than 5 days in one of the weeks and were discarded from the affected weeks. European stations which could not be connected to other stations with baselines shorter than $750 \mathrm{~km}$ were rejected. Another small group of stations recorded complete GPS/GLONASS data sets, and almost all GPS carrier-phase ambiguities could be fixed to integer values, but most GLONASS ambiguities could not be fixed. The Swedish station SPT0 at Borås belongs to this group. It is equipped with the same type of receiver as the Swedish station ONSA at Onsala. While the inter-frequency biases of ONSA have anomalous values (see below), they show the typical linear frequency-dependence and were therefore able to be estimated. The functional model as described above (Eq. 2) seems not to be appropriate for SPT0 and thus the station was rejected.

Between GPS weeks 1570 and 1599 receiver type changes were performed at two stations (Bulgarian station SOFI at Sofia and Austrian station TRF2 at Pernitz). Figure 2 shows two types of receivers for these two stations. Since not all stations and receivers of GPS week 1570 were also present in GPS week 1599, the overall number of individual receivers amounts to 133. Data from 116 receivers were available in both weeks.

At one station (German station LEIJ at Leipzig), the antenna type was changed. It is known that antennas can 
Table 1 GPS/GLONASS

receiver types and numbers of individual receivers used in this study

\begin{tabular}{|c|c|c|c|c|}
\hline \multirow[t]{2}{*}{ Manufacturer } & \multirow[t]{2}{*}{ Receiver type } & \multicolumn{2}{|c|}{ Number of stations } & \multirow[t]{2}{*}{ In Figure 2: Panel (symbol) } \\
\hline & & GPS Week 1570 & GPS Week 1599 & \\
\hline Ashtech (old) & $\mathrm{Z}-18$ & 3 & 3 & $4(\mathrm{~A})$ \\
\hline Ashtech (new) & Pro Flex 500 CORS & - & 2 & $4(5)$ \\
\hline \multirow[t]{2}{*}{ Javad } & TR-G3T Delta & 2 & 1 & $1(8)$ \\
\hline & TR-G3TH Delta & - & 1 & $1(8)$ \\
\hline \multirow[t]{4}{*}{ JPS } & E GGD & 3 & 3 & $1(1)$ \\
\hline & E GGDT & 2 & 3 & $1(2)$ \\
\hline & Eurocard & 1 & 1 & $1(4)$ \\
\hline & Legacy & 13 & 14 & $1(3)$ \\
\hline \multirow[t]{2}{*}{ Leica } & GRX1200+GNSS & 7 & 10 & $2(2)$ \\
\hline & GRX1200 GG PRO & 43 & 44 & $2(1)$ \\
\hline Novatel & OEMV-3 & 3 & 3 & $2(\mathrm{~N})$ \\
\hline Septentrio & PolaRx3eTR & - & 1 & $4(S)$ \\
\hline \multirow[t]{5}{*}{ TPS } & E GGD & 8 & 6 & $1(1)$ \\
\hline & Eurocard & 1 & 1 & $1(4)$ \\
\hline & Odyssey E & 4 & 4 & $1(5)$ \\
\hline & GB-1000 & 4 & 4 & $1(6)$ \\
\hline & NET-G3 & 4 & 4 & $1(7)$ \\
\hline \multirow[t]{2}{*}{ Trimble } & NetR5 & 20 & 22 & $3(5)$ \\
\hline & NetR8 & 1 & 1 & $3(8)$ \\
\hline Sum & - & 119 & 128 & - \\
\hline
\end{tabular}

have a small effect on the GLONASS inter-frequency biases (Wanninger and Wallstab-Freitag 2007) but this aspect is not dealt with in this study.

\section{Data processing}

Inter-frequency biases were determined in baseline-mode using the author's baseline processing-engine Wa1. The combined GPS/GLONASS data processing is based on Eqs. 1 and 2. Standard procedures for precise baseline processing were followed: daily baseline solutions with ambiguity fixing, introduction of IGS satellite and receiver antenna corrections (Kouba 2009), elevation mask of $10^{\circ}$, and restriction to baselines with at least $18 \mathrm{~h}$ of common observations. In order to reduce the influence of those errors which affect baseline solutions depending on the baseline length, the data processing was performed using precise IGS orbits (Kouba 2009), IGS ionospheric grid models (global ionospheric maps-GIM), and estimation of tropospheric zenith delays for baselines longer than $10 \mathrm{~km}$. The iterative algorithm for ambiguity resolution of Wa1 is based on fixing the ambiguities of the widelane linear combination $\mathrm{L}_{\mathrm{W}}$ (wavelength $\lambda_{\mathrm{W}} 84 \cdots 86 \mathrm{~cm}$ ) and of the ionosphere-free linear combination (wavelength $\lambda_{0} \sim$ $11 \mathrm{~cm})$.
Successful and reliable GLONASS ambiguity fixing in mixed baselines can only be achieved if a priori values of the inter-frequency biases are introduced. The GLONASS ambiguity fixing percentage drops considerably to levels of just $30-50 \%$, if larger a priori value differences $($ e.g. $>1 \mathrm{~cm})$ are ignored in mixed baselines. In our data processing, either the values published by Wanninger and Wallstab-Freitag (2007) were taken or, if a newer receiver type was involved, new sets of a priori values were determined in a pre-processing step.

With 2 weeks of observation data and baseline processing according to the baseline selection shown in Fig. 1, 4470 baseline solutions were computed. The baseline results consist of coordinates, tropospheric zenith delays, estimated GLONASS inter-frequency biases, stochastic information in the form of a variance-covariance matrix, and additional statistical information. $79(1.8 \%)$ of the baseline results were discarded since the GLONASS ambiguity fixing percentage fell below a threshold of $90 \%$ or the standard deviation of unit weight exceeded a threshold of $1.6 \mathrm{~cm}$. Of the remaining 4,391 baselines solutions, only the estimated GLONASS inter-frequency biases in L1, L2 and in the ionosphere-free linear combination L0 were used in further processing steps.

Within each of the 2 weeks, standard deviations of the estimated daily inter-frequency biases were computed to show the repeatability of these estimates. A detailed analysis revealed that standard deviations increased when certain 


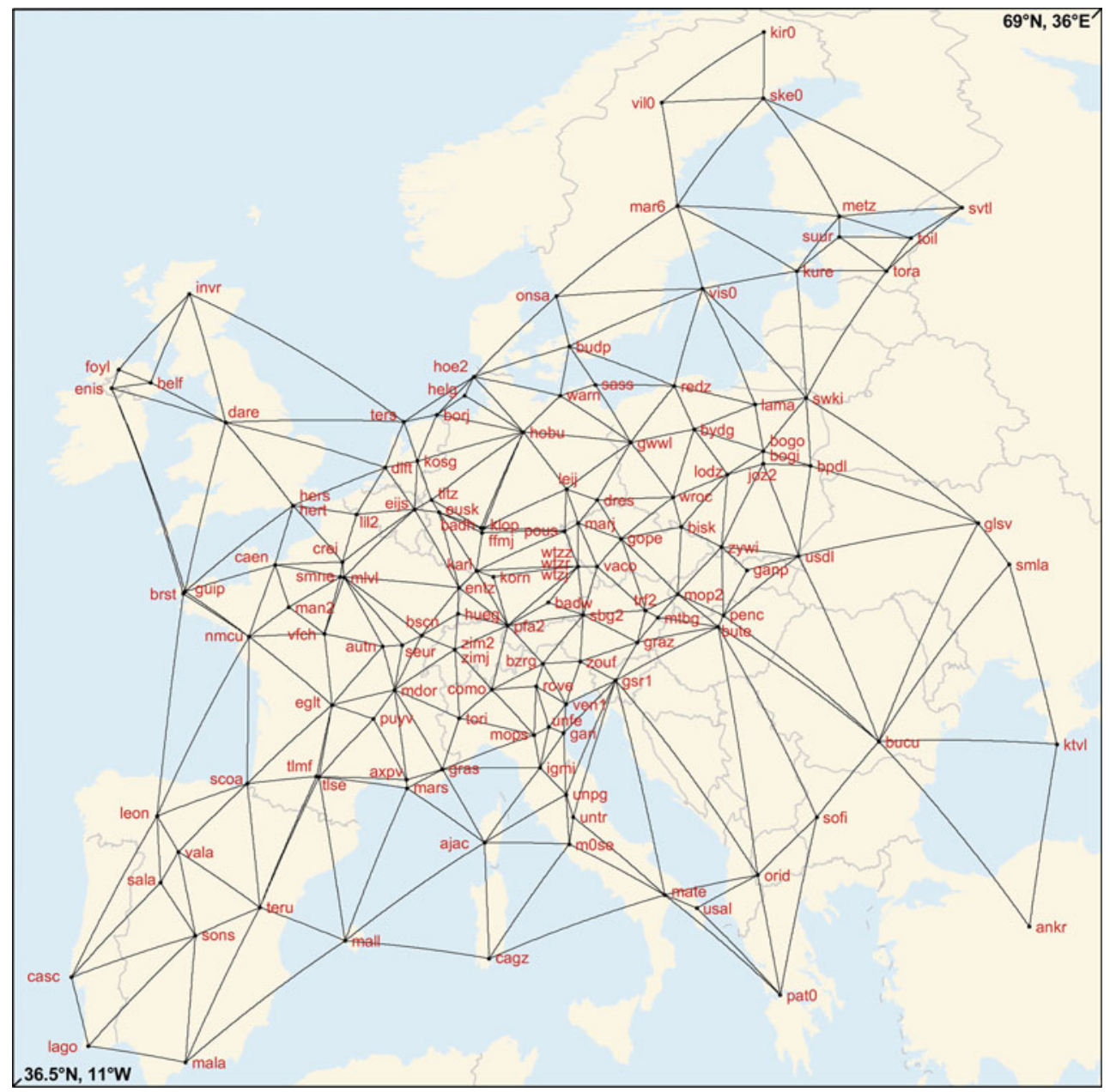

Fig. 1 Selected GPS/GLONASS reference stations in Europe and baselines used in the data processing

stations were involved. Among these are all stations equipped with Ashtech Z-18 receivers, the oldest of all GPS/GLONASS receiver types still in use. These receivers are not able to track GLONASS satellite signals with channel number zero or with negative channel numbers. As a consequence, these receivers collect only about half the amount of GLONASS observations other receivers do and the maximum channel number difference is just 5 as compared to 13 with other receivers. Thus, the accuracy of the estimated inter-frequency biases is much lower and the day-to-day repeatability much worse.

Individual stations, which lead to worse baseline repeatabilities, include the Italian station TORI at Torino and the Greek station PAT0 at Patras. The repeatability of the estimates of GLONASS inter-frequency biases $\left(c_{0} \Delta \delta h\right)$ of these stations are shown in separate panels of Fig. 3.

In general, the repeatability of the bias estimates (lower panels of Fig. 3) shows baseline length dependence on L1, even more pronounced on L2 but no such dependence in the ionosphere-free linear combination L0. This characteristic proves that remaining ionospheric effects influence the results for L1 and L2. Nevertheless, repeatability is always better than a few millimeters and, if the outliers shown in the upper panels of Fig. 3 are ignored, even in L2 the repeatability is better than $1 \mathrm{~mm}$ for $93 \%$ of all baselines.

Baseline results include estimates of the differences of GLONASS inter-frequency biases between pairs of individual receivers. Even with a large set of baseline results as computed in this study, no absolute inter-frequency biases can be obtained. Thus, we can choose freely how to set the absolute level of the inter-frequency biases. It is important, however, that the same absolute level is realized in both selected weeks so that values from different weeks can be directly compared.

In a first adjustment of the inter-frequency biases of all daily baseline solutions, it was found that the variations among individual receivers of the same type can differ considerably. These variations may be caused by the receivers themselves but also by different antenna types or by other differences in the station hardware. Smallest variations were observed for receiver types JPS Legacy, Leica GRX1200 GG PRO, and Trimble NetR5 (cf. Fig. 5). 

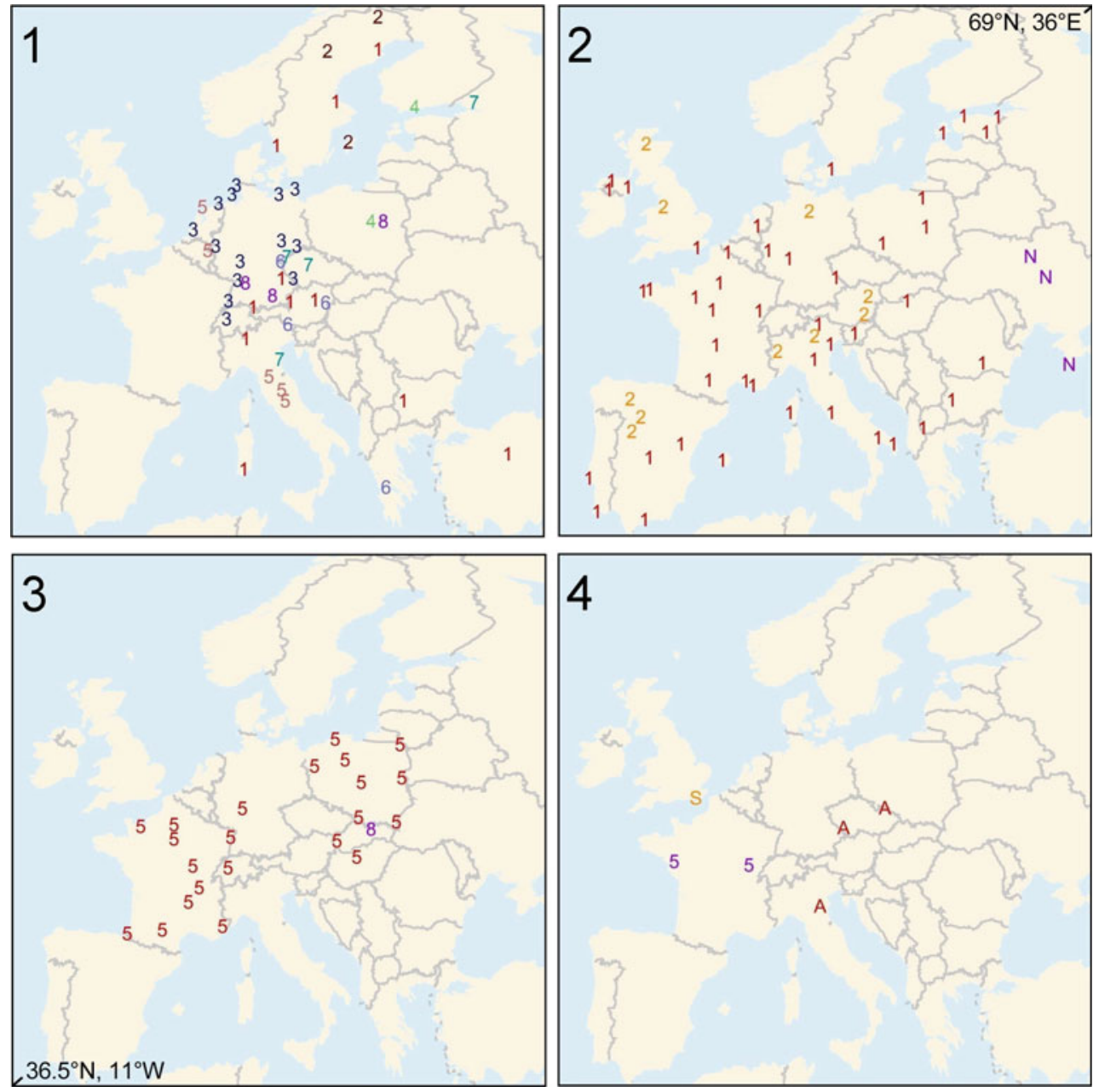

Fig. 2 Distribution of GPS/GLONASS equipment sorted by manufacturers. Panel 1 JPS, TPS, Javad; Panel 2 Leica, Novatel; Panel 3 Trimble; Panel 4 Ashtech (old), Ashtech (new), and Septentrio. The symbols indicate receiver types and are explained in Table 1
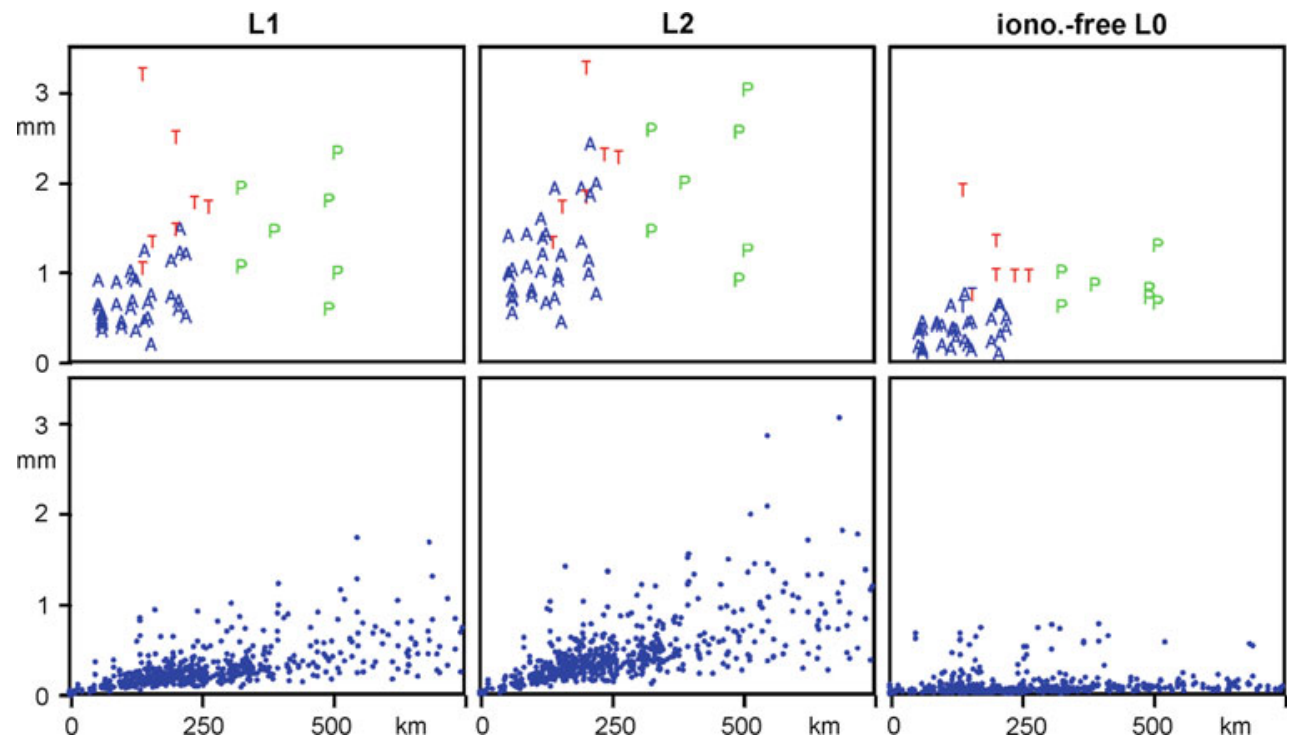

Fig. 3 Baseline length dependence of the repeatability (RMS values) of estimated GLONASS inter-frequency biases in L1, L2, and the ionospheric-free linear combination L0. Upper panels baselines with at least

one station being equipped with an Ashtech Z-18 receiver $(A)$, baselines including the station TORI $(T)$, or the station PAT0 $(P)$. Lower panels results of all other baselines 
The JPS Legacy receivers belong to the group of older receivers together with other JPS and TPS receivers and the Ashtech Z-18. All these older receivers have similar GLONASS inter-frequency biases. Other manufacturers that released their GPS/GLONASS equipment from 2006 onward did not bring the inter-frequency biases of their receivers in agreement with the biases of the already existing receivers. Due to this historical development, it was decided to use the 12 JPS Legacy receivers present in both weeks to set the absolute level of the biases. The adjustment of all baseline solutions was repeated with the constraint that the mean bias value of the selected JPS Legacy receivers equals zero. As a result, the absolute bias values presented here for all older receivers are fairly small.

\section{Results}

Sets of GLONASS inter-frequency biases were computed from all baseline solutions for GPS weeks 1570 and 1599. As described above, one constraint was introduced to obtain absolute values: the average biases of 12 JPS Legacy receivers were set to zero. Three different solutions were calculated: for L1, L2, and for the ionosphere-free linear combination L0. All bias values mentioned in this chapter refer to delay differences between adjacent frequency channels. The values are given in units of meters, i.e. $\Delta \delta h$ of Eq. 2 was multiplied by the speed of light in a vacuum $c_{0}$.

The comparison of the L1 and the L2 results shows that in general similar biases exist in the two frequency bands (Fig. 4). But, there is one distinct exception: the JPS E GGD receiver at the Swedish station ONSA, which we look at in more detail below. Some stations have slightly different L1/L2 biases, such as the Javad TR-G3T Delta receiver at the Polish station BOGI at Borowa Gora. In general, large bias differences exist between receivers from different manufacturers. Five manufacturer groups can be distinguished: Trimble, old Ashtech/Javad/JPS/TPS, new Ashtech, Leica/Novatel, and Septentrio. Please note that there is only a single Septentrio receiver which contributed to the data set.

Bias differences between receivers from different manufacturers can reach up to $5 \mathrm{~cm}(0.2 \mathrm{~ns})$ for adjacent frequencies and thus up to $73 \mathrm{~cm}(2.4 \mathrm{~ns})$ for the complete L1 or L2 frequency bands. When relating this to the signal wavelengths of about 19 or $24 \mathrm{~cm}$ or the wavelengths of often used linear combinations e.g. 84 or $11 \mathrm{~cm}$, it becomes obvious that reliable ambiguity fixing requires accurate a priori bias corrections.

When forming the ionosphere-free linear combination L0 similar values are obtained as long as the L1/L2 bias differences are small. Figure 5 shows the L0 results sorted by manufacturers. Here, the sample sizes become more visible and also the variations among receivers of the same

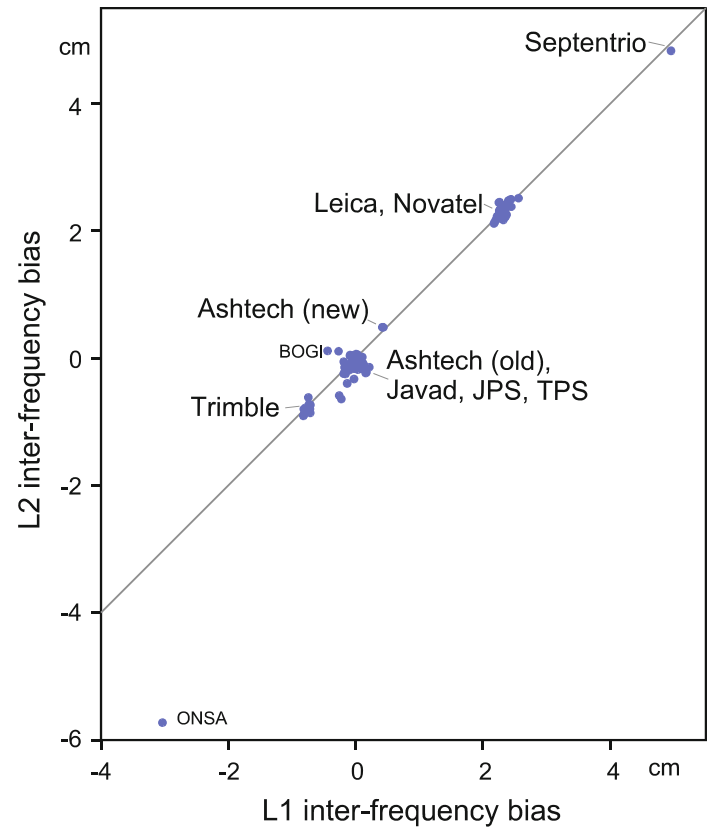

Fig. 4 Comparison of GLONASS L1 and L2 inter-frequency biases of 133 individual receivers

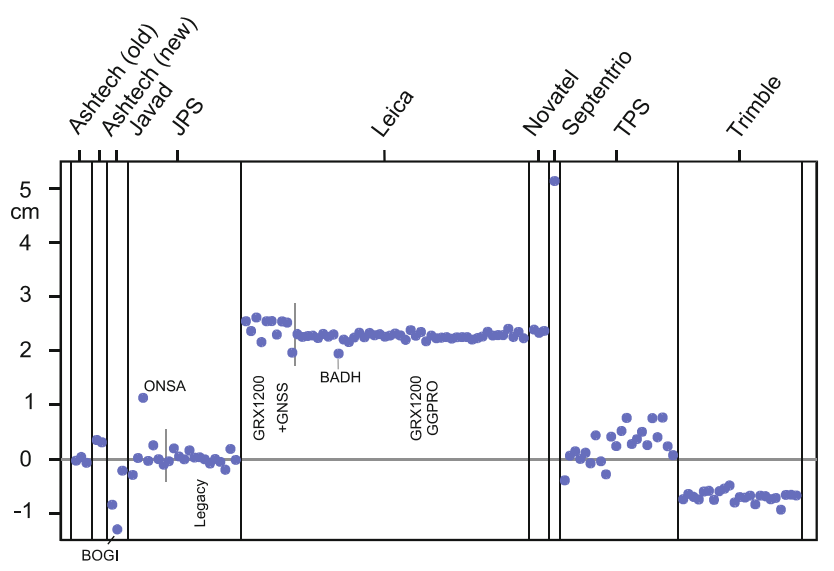

Fig. 5 GLONASS inter-frequency biases in the ionosphere-free linear combination L0 of 133 individual receivers grouped by manufacturers and receiver types

type. The good agreement within the groups of JPS Legacy, Leica GRX1200 GG PRO, and Trimble NetR5 receivers has already been mentioned above. Larger variations are noticeable for Javad and TPS receivers. A closer look at the one outlier among the inter-channel biases of 44 Leica GRX1200 GG PRO receivers reveals that it belongs to the German station BADH at Bad Homburg. Only here a GPS/GLONASS receiver is equipped with an antenna of type TRM41249.00 NONE (Trimble Zephyr Geodetic) which is a GPS antenna not originally intended by the manufacturer for GLONASS signal reception. It can be speculated that this offset of the GLONASS inter-channel biases is caused by the antenna.

In order to gather more information on the GLONASS inter-frequency biases for the JPS E GGD receiver at the 


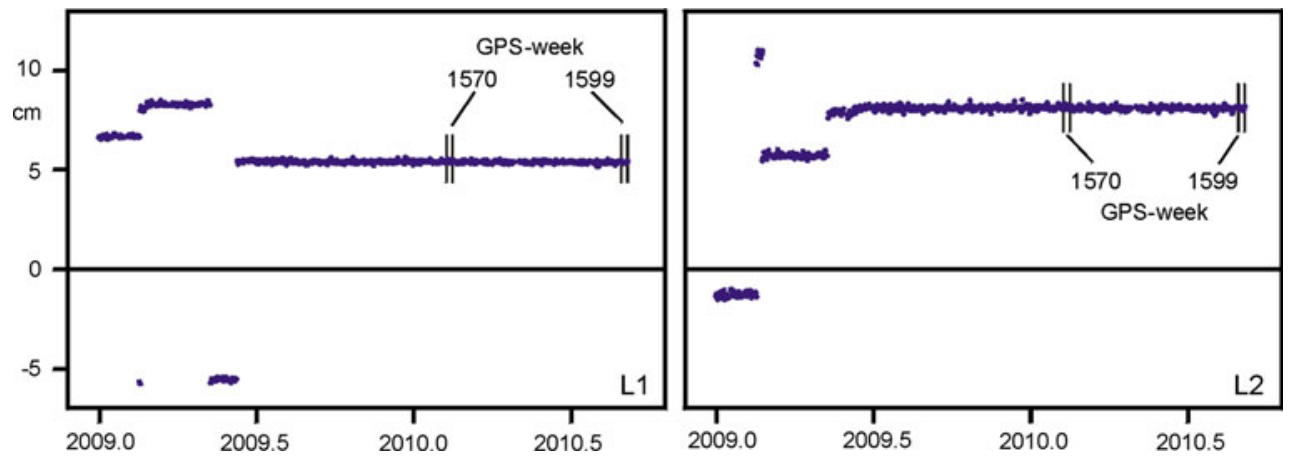

Fig. 6 Daily estimates of L1 and L2 GLONASS inter-frequency bias differences in the baseline BUDP-ONSA for more than 1.5 years. The discontinuities in year 2009 are caused by the receiver at station ONSA

Swedish station ONSA, daily bias estimates were computed for the $188 \mathrm{~km}$ long baseline from the Danish station BUDP at Kobenhavn equipped with a Leica GRX1200 GG PRO receiver to ONSA. Fig. 6 shows these daily L1 and L2 estimates from day of year (DoY) 1 in 2009 (1 January 2009) until DoY 250 in 2010 (7 September 2010). The time series reveal several jumps in the GLONASS inter-frequency biases over the first half of 2009, but stable biases afterwards. It could be confirmed by the results of other baselines that these jumps are caused by the receiving equipment at ONSA. The jumps occur at the same times as gaps in the data from ONSA, probably caused by receiver failures. Thus, it can be speculated that the restart of the receiver causes an internal re-calibration of the GLONASS inter-frequency biases. Unfortunately, the Onsala Space Observatory was not able to confirm or to disprove receiver failures or re-starts of the receiver on these dates. Please note that the time series of Fig. 6 do not reveal any variations which could be attributed to temperature effects, e.g. due to the annual temperature cycle.

In a further step, independent solutions of the GLONASS inter-frequency biases were computed for each of the 2 weeks. Figure 7 shows the comparison of the L0 biases of the 2 weeks. Here, only results for those receivers present in both weeks are shown. For this reason the figure does not include the single Septentrio receiver which had not been installed during the first week. Just one of the three Javad receivers, the one at BOGI, was available in both weeks.

In general, GLONASS inter-frequency biases for the ionosphere-free linear combination L0 do not change considerably over this 6-month period separating the two test weeks. Bias differences between the 2 weeks were determined to be smaller than $2 \mathrm{~mm}$ for $92 \%$ of the receivers.

Three differences exceed $3 \mathrm{~mm}$ reaching 6.0, 6.1, and $7.4 \mathrm{~mm}$ for the TPS receivers at the Dutch stations TERS at West-Terschelling and EIJS at Eijsden, and at the Italian station COMO at Como, respectively. The biases of these three receivers were analyzed in more detail by processing daily baseline solutions from DoY 1 to DoY 250 in 2010.

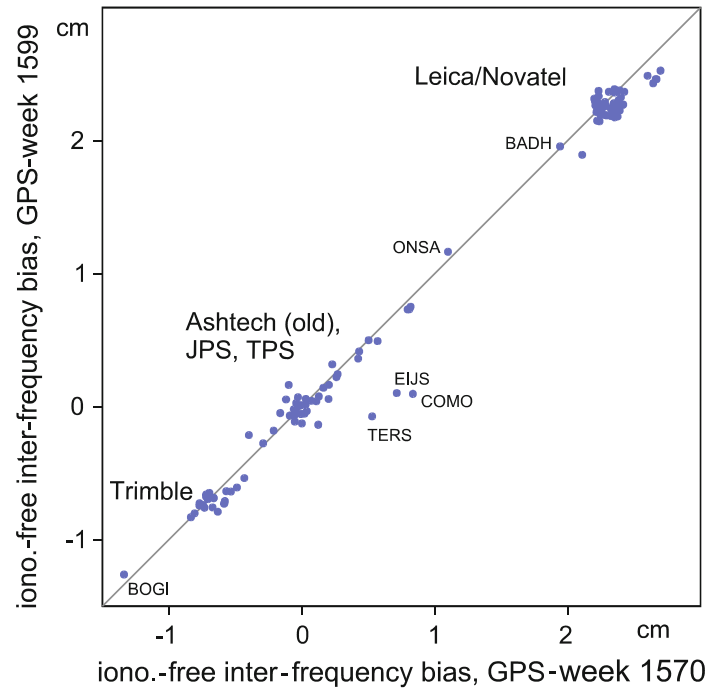

Fig. 7 Comparison of GLONASS inter-frequency biases in the ionosphere-free linear combination L0 of 116 individual receivers present in both weeks

The second stations in each baseline were selected according to their limited geographical distance from the station in question, data availability, and the stability of their GLONASS inter-frequency biases. The results are shown in Fig. 8, with two panels for each baseline, the first for L1 and the second for L2. Day-to-day variation of the determined biases is larger for L2 than for L1 and depends very much on the baseline length. A significant portion of it is caused by remaining ionospheric effects (cf. Fig. 3). The jumps in the GLONASS inter-frequency biases occur on L1 only, while L2 is free of any jumps. The jumps on $\mathrm{L} 1$ have sizes of about $2 \mathrm{~mm}$ and were amplified by forming the ionosphere-free linear combination L0 whose results are shown in Fig. 7.

The three affected TPS receivers underwent changes in their firmware. The dates of the firmware changes are marked in Fig. 8. For the first two baselines, the dates correspond to the dates of the jumps in the GLONASS inter-frequency 

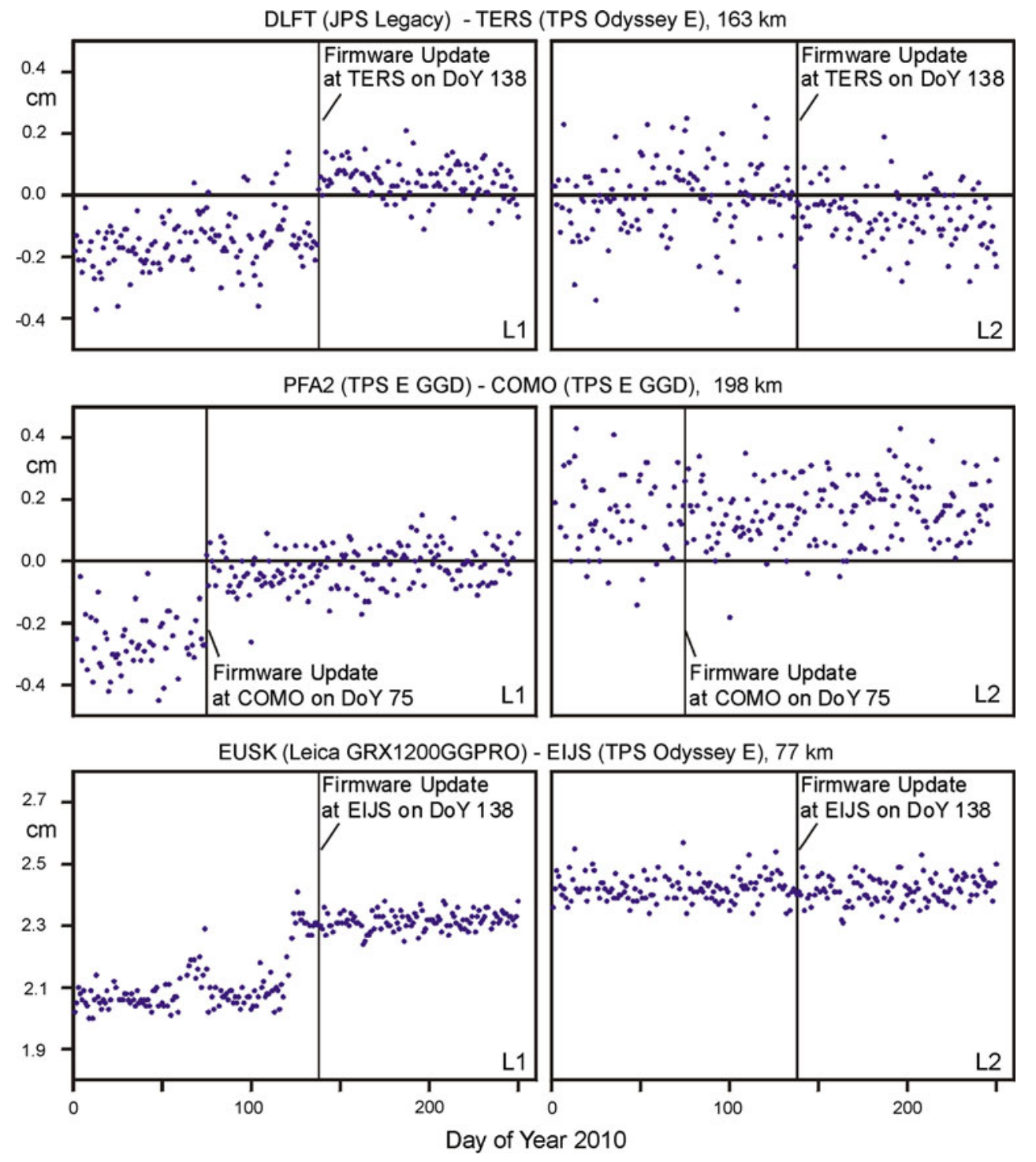

Fig. 8 Daily estimates of L1 and L2 GLONASS inter-frequency bias differences in three baselines. Firmware changes took place in the receivers of the second station of each baseline

time series. For the third baseline however, the jump occurred 20 days earlier after a short observation gap. Our conclusion is that the firmware changes are not responsible for the modification of the L1 GLONASS inter-frequency biases and that this is instead due to a receiver restart. Again, we speculate that such a restart of a TPS receiver probably causes an internal recalibration of the inter-frequency biases.

The two sample weeks had been selected with a time offset of about 6 months to try to identify seasonal temperature effects on the estimated inter-channel biases. However, as Fig. 7 reveals, no large differences can be found which could be attributed to temperature changes. Furthermore, no such seasonal effects are detectable in the long time series of inter-channel biases presented in Figs. 6 and 8. Thus, we were not able to find any indications for significant seasonal temperature dependencies of the GLONASS inter-frequency biases. The reason for this may lie in the internal temperature stabilization of the receivers.

\section{Conclusion}

The determination of GLONASS carrier-phase inter-frequency biases for 133 individual GPS/GLONASS receivers installed at continuously operating stations in Europe reveals that large bias differences exist among equipment from different manufacturers. In general, the biases can be modeled as linear functions of frequency. They are similar for L1 and L2 and also for receivers of the same type. 
Table 2 Proposed a priori corrections of L1 and L2 GLONASS interfrequency biases for receivers of nine different manufacturers

\begin{tabular}{lc}
\hline Receiver manufacturer & A priori corrections $(\mathrm{cm})$ \\
\hline Trimble & -0.7 \\
Ashtech (old), Javad, JPS, TPS & 0.0 \\
Ashtech (new) & 0.4 \\
Leica, Novatel & 2.3 \\
Septentrio & 4.9 \\
\hline
\end{tabular}

The biases typically seem to be stable over time, but for some receiver types a restart of the receiver may cause small modifications of the biases. One of the tested receivers, the JPS E GGD receiver at the Swedish station ONSA, behaves differently. But even here, the inter-frequency biases are linear functions of frequency. No indications were found that the biases are sensitive to temperature changes.

The correct and reliable fixing of GLONASS carrier-phase ambiguities requires a priori correction of the inter-frequency biases. Table 2 summarizes the results of this study in the form of such a priori corrections for five groups of manufacturers. They are recommended for use when ambiguity fixing in baselines with receivers from manufacturers of different groups. After successful ambiguity fixing, differences of individual receiver inter-frequency biases must be estimated for each baseline.

Acknowledgments The author kindly acknowledges the help of Susanne Haase with the pre-processing of the observation data, and the support by Dorothee Weniger who prepared Figs. 1 and 2. The following institutions are acknowledged for providing GPS/GLONASS observation data: EUREF Permanent Network (EPN), International GNSS Service (IGS), TERIA Network, France, Bavarian Commission for International Geodesy, Munich, Germany, and Institute of Communications and Navigation of the German Aerospace Center, Neustrelitz, Germany. Last but not least, the author acknowledges helpful comments by P. Willis and three anonymous reviewers of the manuscript.

Open Access This article is distributed under the terms of the Creative Commons Attribution Noncommercial License which permits any noncommercial use, distribution, and reproduction in any medium, provided the original author(s) and source are credited.

\section{References}

Bruyninx C, Becker M, Stangl G (2001) Regional densification of the IGS in Europe using the EUREF permanent GPS network (EPN). Phys Chem Earth 26(6-8): 531-538. doi:10.1016/ S1464-1895(01)00096-5

Dow JM, Neilan RE, Rizos C (2009) The International GNSS Service in a changing landscape of Global Navigation Satellite Systems. J Geod 83: 191-198. doi:10.1007/s00190-008-0300-3

Hofmann-Wellenhof B, Lichtenegger H, Wasle E (2008) GNSSGlobal Navigation Satellite Systems. Springer, Wien

ICD (2008) GLONASS Interface Control Document, edn 5.1, Russian Institute of Space Device Engineering

Kouba J (2009) A Guide to Using International GNSS Service (IGS) Products. http://igscb.jpl.nasa.gov/igscb/resource/ pubs/UsingIGSProductsVer21.pdf

Kozlov D, Tkachenko M, Tochilin A (2000) Statistical characterization of hardware biases in GPS+GLONASS receivers. In: Proceedings of ION GPS 2000, pp 817-826

Leick A (2004) GPS Satellite Surveying, 3rd edn. Wiley, Hoboken

Pratt M, Burke B, Misra, P (1998) Single-epoch integer ambiguity resolution with GPS-GLONASS L1-L2 Data. In: Proceedings of ION GPS-98, pp 389-398

Revnivykh S (2010) GLONASS Status and Progress. In: Proceedings of ION GNSS 2010, pp 609-633

Wanninger L, Wallstab-Freitag S (2007) Combined processing of GPS, GLONASS, and SBAS code phase and carrier phase measurements. In: Proceedings of ION GNSS 2007, pp 866-875

Yamanda H, Takasu T, Kubo N, Yasuda A (2010) Evaluation and calibration of receiver inter-channel biases for RTK-GPS/GLONASS. In: Proceedings of ION GNSS 2010, pp 1580-1587

Zinoviev AE (2005) Using GLONASS in combined GNSS receivers: current status. In: Proceedings of ION GNSS 2005, pp 1046-1057

Zinoviev AE, Veitsel AV, Dolgin DA (2009) Renovated GLONASS: improved performances of GNSS receivers. In: Proceedings of ION GNSS 2009, pp 3271-3277 